Reprod. Nutr. Dévelop., 1987, 27 (2 A), 399-406.

\title{
Effet d'une carence hydrique prolongée sur la croissance pondérale et sur le métabolisme hydrique chez un rongeur Gerbillidé (Meriones shawi shawi)
}

\author{
Malika SAHNI, Jacqueline PEIGNOUX-DEVILLE, M. ZNARI, Evelyne LOPEZ, \\ F. LACHIVER
}

avec la collaboration technique de Francine LALLIER, Elizabeth MARTELLY et Bernadette VIDAL

Laboratoire de Physiologie Générale et Comparée du Muséum national d'Histoire naturelle et Laboratoire d'Endocrinologie Comparée associé au C.N.R.S.,

7, rue Cuvier, 75231 Paris Cedex 05.

Summary. Effect of prolonged water deprivation on weight gain and water metabolism in sub-desert rodents (Meriones shawi shawi).

The effect of prolonged water deprivation, applied progressively since weaning, was studied on body weight in growing sub-desert rodents (Meriones shawi). Then, water metabolism, food consumption and kidney weight were measured in these adults animals.

During partial water deprivation the weight curve was similar to that of controls, whereas with total water deprivation three different stages were noticed : a decrease, a stabilization and an increase in body weight as a function of age.

At the end of the experiment, the body water volume was similar in dehydrated and in control animals. However, in water deprived animals, there was a lower rate of water influx and efflux compared to controls. Their food consumption was two-fold that of controls. Dehydrated meriones showed a significant increase in kidney weight as compared to controls.

\section{Introduction.}

Dans certains biotopes, le manque d'eau peut constituer un facteur limitant pour la croissance et la survie des espèces qui les peuplent. En effet, les mammifères non désertiques ne peuvent résister que quelques jours à une carence hydrique prolongée; ainsi, le rat meurt au bout de deux semaines, alors que la mérione montre, dans les mêmes conditions, une baisse puis une stabilisation de poids (Rouffignac et Morel, 1965). Les rongeurs vivant dans les zones arides parviennent à équilibrer leur balance hydrique en mettant en jeu un certain nombre de facteurs écologiques (Petter, 1961) et de mécanismes physiologiques, par exemple des modifications de la fonction rénale (Schmidt-Nielsen, 1964 ; Ivanova et al., 1980), ou l'augmentation des taux d'hormones antidiurétiques plasmatiques et hypophysaires (Baddouri et al., 1984). Peu d'auteurs ont étudié les effets d'une privation d'eau sur la croissance et le métabolisme hydrique. Seul le Mouton chokla, intéressant par sa valeur économique en Inde, a. fait l'objet de certains 
travaux (Taneja, 1965 ; Purohit et al., 1972 ; More et Sahni, 1979). Ces derniers ont mis en évidence chez le mouton chokla une perte de poids de $16,4 \%$ en été et une économie d'eau de 30 à $40 \%$.

Notre étude a porté sur un rongeur semi-désertique de la famille des Gerbillidés, une mérione (Meriones shawi) qui, par son comportement écologique (Petter, 1961 ; Lachiver et al., 1978) et la mise en œuvre de mécanismes physiologiques (Petter et Lachiver, 1983) semble être bien adaptée à l'économie d'eau.

Nous avons recherché, d'une part l'effet d'une carence hydrique prolongée, imposée dès le sevrage, sur l'évolution du poids corporel, d'autre part les modifications du poids du rein, du métabolisme hydrique et de la consommation alimentaire chez ces animaux au stade adulte.

\section{Matériel et méthodes.}

Les mériones utilisées proviennent d'un élevage développé au laboratoire à partir d'individus capturés en Tunisie. Les animaux (génération F2) sont sevrés 25 jours après la naissance (poids moyen : $23,4 \pm 0,4 \mathrm{~g}$ ), isolés en cages individuelles $(20 \times 19 \times 15 \mathrm{~cm})$ et placés dans une chambre climatique $\left(\mathrm{T}^{\circ}=25^{\circ} \mathrm{C}\right.$; $\mathrm{HR}=65 \%$; photopériode : 12L-12D). Au fur et à mesure des naissances, chaque fratrie est soumise à l'un ou l'autre des régimes décrits ci-dessous jusqu'à obtention de deux groupes de 20 animaux chacun. Tous les animaux sont sacrifiés en même temps à un âge compris entre 90 et 120 jours. Les animaux témoins ont reçu un régime alimentaire riche en eau constitué de biscuits pour rats (aliment complet stérilisable) et de pommes ad libitum, les pommes étant leur unique source d'eau, jusqu'au sacrifice. Pour les animaux " déshydratés " $\left({ }^{*}\right)$, l'apport en pommes a été réduit dès le sevrage de façon à fournir approximativement la moitié des besoins normaux en eau de l'animal (soit $4 \mathrm{~g}$ ) durant 21 jours. Ensuite, les animaux reçoivent le quart (soit $2 \mathrm{~g}$ ) de leurs besoins pendant 14 jours, puis l'apport en pommes est supprimé jusqu'à la fin de l'expérience (fig. 1). A ce moment le régime pauvre en eau est constitué uniquement de biscuits. Les besoins hydriques ont été calculés à partir des valeurs de flux entrant d'eau déterminées chez la mérione adulte par Bradshaw et al. (1976) dans les conditions naturelles. Chaque animal recevant l'un ou l'autre des deux régimes est pesé à $0,1 \mathrm{~g}$ près, 3 fois par semaine jusqu'à la fin de l'expérience. Nous avons choisi de représenter les courbes de poids d'une fratrie dans chacun des régimes, celles-ci étant représentatives de l'évolution du poids corporel dans toutes les autres fratries.

Une semaine avant le sacrifice, les flux d'eau entrant et sortant sont déterminés à l'aide d'eau tritiée ( ${ }^{3} \mathrm{HHO}$ ) selon Lifson et Mc Clintock (1966). La consommation alimentaire en pommes et en biscuits est estimée à l'aide de sodium-22 (22 Na) par la méthode de Green (1978) appliquée aux rongeurs par Znari (1983). La radioactivité plasmatique en ${ }^{3} \mathrm{H}$ et ${ }^{22} \mathrm{Na}$ est mesurée, sur $100 \mu \mathrm{l}$ de plasma, au spectromètre à scintillation liquide (Intertechnique ABAC SL 4000), le rendement

(*) Le terme déshydraté est utilisé pour : soumis progressivement à carence hydrique. 
des mesures étant déterminé par étalonnage externe. Le sodium dans les aliments et dans le plasma est dosé par spectrophotométrie de flamme (Eppendorf). Le calcul des pools et des flux entrant et sortant d'eau et de sodium est effectué selon la formule de Lifson et Mc Clintock (1966) reprises par Nagy et Costa (1980). La même formule de Nagy est utilisée pour calculer les flux entrant et sortant de $\mathrm{Na}$ en volume de plasma équivalent. Connaissant la natrémie, ces flux sont calculés en mmol de $\mathrm{Na}$. Les quantités respectives de biscuits et de pommes ingérées par les animaux témoins et de biscuits par les "déshydratés " sont déterminées à l'aide des formules données par Znari (1983). Au moment du sacrifice, les reins sont prélevés et pesés.

Les tests statistiques ont été effectués par une analyse de type non paramétrique (test de Fisher). En ce qui concerne le poids du rein, le traitement des valeurs expérimentales a été effectué grâce à un programme de régression permettant de linéariser les relations : poids du rein, poids du corps. Les paramètres des droites de régression ont été calculés avec détermination des coefficients de corrélation entre poids du rein et poids du corps.

\section{Résultats.}

1. Etudes pondérales. - L'analyse de la courbe pondérale des animaux témoins (fig. 1) montre une augmentation puis une stabilisation aux environs de 95 jours.

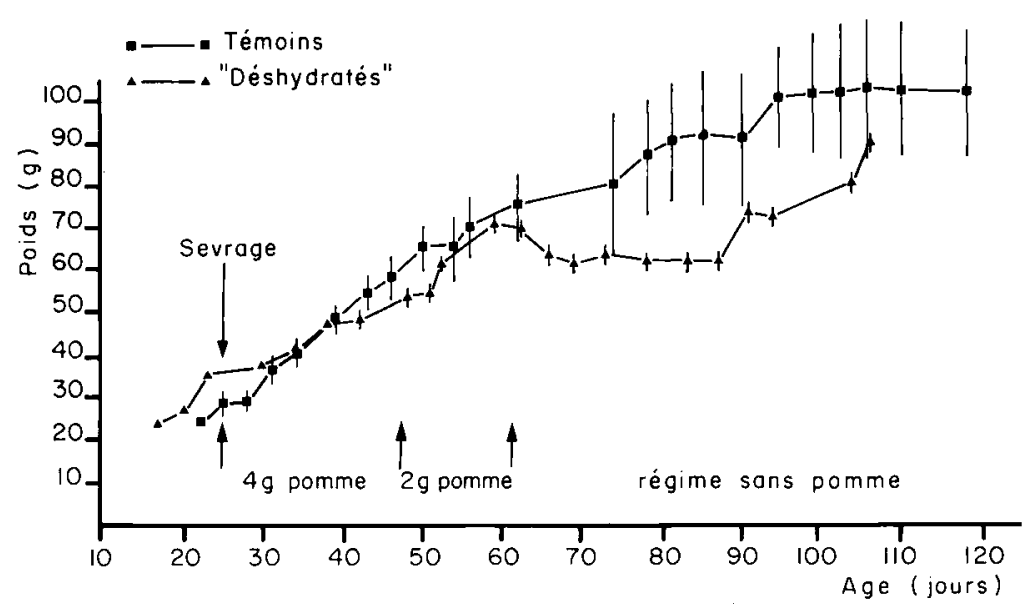

FIG. 1. - Evolution du poids des mériones témoins et "déshydratées " en fonction du temps. Chaque point représente la moyenne de 4 déterminations (correspondant à une fratrie).

La courbe de poids des animaux déshydratés est pratiquement parallèle à celle des témoins jusqu'à l'âge de 62 jours. La carence partielle en eau ne semble pas modifier la vitesse de croissance. La privation totale d'eau entraîne une chute pondérale de $10 \%(p<0,02)$ en 4 jours. On constate ensuite une stabilisation du poids pendant 24 jours suivie d'une augmentation pondérale de $18 \%(p<0,05)$ 
en 4 jours. A ce moment la courbe de poids des mériones déshydratées tend à rejoindre celle des animaux témoins.

Les capacités de résistance à un régime déshydraté sont très variables d'un animal à l'autre. Le taux de mortalité qui est de $10 \%$ chez les témoins est nettement plus élevé chez les animaux déshydratés (30\%). L'étude des courbes de poids du rein en fonction du poids corporel met en évidence dans chacun des groupes (témoins et déshydratés), une bonne corrélation entre le poids de cet organe et le poids du corps, avec des coefficients de corrélation hautement significatifs, respectivement égaux à 0,85 et 0,91 (fig. 2). Les calculs statistiques montrent que les pentes des droites $(8,39 \pm 1,25$ et $8,50 \pm 1,05)$ ne sont pas significativement différentes dans les deux groupes d'animaux, mais pour un poids corporel donné $(X)$, le poids rénal (Y1) chez la mérione déshydratée est en moyenne 1,5 fois supérieur à celui de l'animal témoin (Y2).

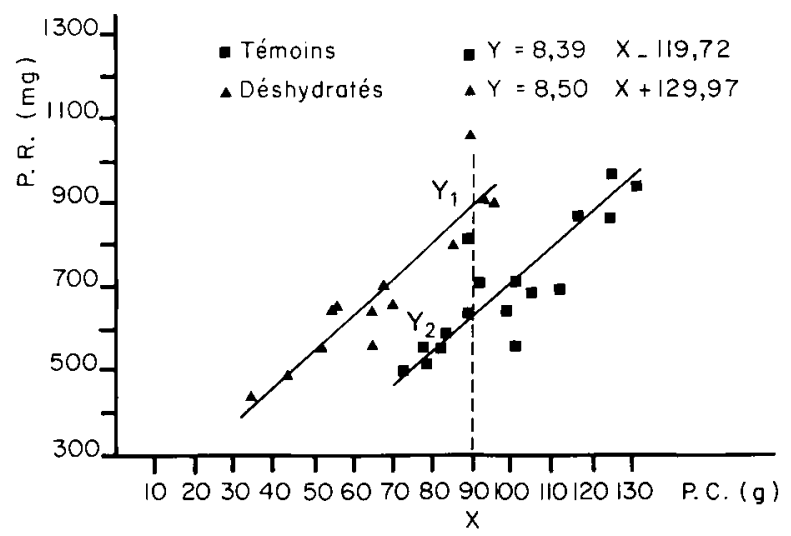

FIG. 2. - Poids du rein en fonction du poids du corps des merriones témoins et " déshydratées » en fin d'expérience. Chaque point ( $a$ ) représente la moyennę de 17 déterminations. Chaque point (A) représente la moyenne de 13 déterminations.

TABLEAU 1

Volume d'eau corporelle. Flux d'eau entrant et sortant chez les mériones témoins et "déshydratées ».

\begin{tabular}{|c|c|c|c|c|c|c|}
\hline \multirow{3}{*}{ Régimes } & \multirow{3}{*}{$\begin{array}{l}\text { Poids } \\
\text { (g) }\end{array}$} & \multirow{3}{*}{$\begin{array}{c}\text { Volume } \\
\text { d'eau } \\
\text { corporelle } \\
(\%)\end{array}$} & \multicolumn{4}{|c|}{ Flux entrant $(\mathrm{Fe})$ et sortant (Fs) } \\
\hline & & & \multicolumn{2}{|c|}{$\mathrm{ml} \mathrm{j}^{-1}$} & \multicolumn{2}{|c|}{$\mathrm{ml} \mathrm{kg} \mathrm{j-1}$} \\
\hline & & & $\mathrm{Fe}$ & $\mathrm{Fs}_{\mathrm{s}}$ & $\mathrm{Fe}$ & Fs \\
\hline $\begin{array}{c}\text { Régime } \\
\text { normal } \\
n=5 \\
(5 Q)\end{array}$ & $85,88 \pm 7,63$ & $78,10 \pm 7,58$ & $21,22 \pm 1,91$ & $21,45 \pm 1,93$ & $247,95 \pm 13,02$ & $249,94 \pm 12,72$ \\
\hline $\begin{array}{c}\text { Régime } \\
\text { déshydraté } \\
n=4 \\
(4 \bigcirc)\end{array}$ & $76,90 \pm 3,17$ & $76,95 \pm 6,45$ & $6,51 \pm 1,51$ & $6,85 \pm 1,08$ & $89,89 \pm 16,83$ & $91,09 \pm 16,67$ \\
\hline
\end{tabular}

$\Delta \mathbf{p}<0,001$ par rapport aux témoins. Les valeurs sont obtenues par la méthode à l'eau tritiée. 
2. Echanges hydriques et sodiques et consommation alimentaire. - Les mesures des échanges hydriques (tabl. 1) ne montrent, chez les mériones déshydratées comparées aux témoins, aucune modification significative du volume relatif d'eau corporelle. Chez les témoins, les valeurs des flux d'entrée (Fe) et de sortie (Fs) d'eau sont identiques, ce qui reflète l'équilibre hydrique chez ces animaux. Chez les déshydratés, on constate que les flux d'eau (Fe) et (Fs) sont diminués et ne représentent que le tiers de ceux des témoins. Les animaux carencés en eau sont en équilibre hydrique. Les taux de renouvellement d'eau chez les témoins et les déshydratés sont respectivement égaux à $31,98 \%$ et $11,58 \%$ du volume d'eau corporelle renouvelée par jour.

En ce qui concerne les échanges sodiques, les mériones déshydratées, comparées aux témoins, ne montrent aucun changement significatif du pool échangeable du sodium. Les mériones témoins sont en équilibre sodique (Fe de $\mathrm{Na}=\mathrm{Fs}$ de $\mathrm{Na}$ ). Chez les mériones déshydratées on constate une augmentation significative des valeurs des flux entrant et sortant de sodium, le flux net restant nul dans ce groupe (tabl. 2).

TABLEAU 2

Pool échangeable. Flux de sodium entrant et sortant chez les mériones témoins et " déshydratées ».

\begin{tabular}{|c|c|c|c|c|c|c|}
\hline \multirow{3}{*}{ Régimes } & \multirow{3}{*}{$\begin{array}{l}\text { Poids } \\
\text { (g) }\end{array}$} & \multirow{3}{*}{$\begin{array}{c}\text { Pool } \\
\text { échangeable } \\
\text { (mmol) }\end{array}$} & \multicolumn{4}{|c|}{ Flux entrant $(\mathrm{Fe})$ et sortant (Fs) } \\
\hline & & & \multicolumn{2}{|c|}{$\mathrm{mmol} \mathrm{j}^{-1}$} & \multicolumn{2}{|c|}{$\mathrm{mmol} \mathrm{kg} \mathrm{k}^{-1} \mathrm{j}^{-1}$} \\
\hline & & & $\mathrm{Fe}$ & Fs & $\mathrm{Fe}$ & Fs \\
\hline $\begin{array}{c}\text { Régime } \\
\text { normal } \\
n=5 \\
(5 \%)\end{array}$ & $85,88 \pm 7,63$ & $5,24 \pm 1,36$ & $0,24 \pm 0,08$ & $0,24 \pm 0,08$ & $2,64 \pm 0,68$ & $2,65 \pm 0,68$ \\
\hline $\begin{array}{c}\text { Régime } \\
\text { déshydraté } \\
\mathrm{n}=4 \\
(40)\end{array}$ & $76,90 \pm 3,17$ & $6,24 \pm 1,13$ & $0,42 \pm 0,04$ & $0,42 \pm 0,04$ & $5,62 \pm 0,83$ & $5,64 \pm 0,84$ \\
\hline
\end{tabular}

TABLEAU 3

Consommation alimentaire chez les mériones témoins et "déshydratées " en fin d'expérience.

\begin{tabular}{|c|c|c|}
\hline \multirow{2}{*}{ Régimes } & \multicolumn{2}{|c|}{ Consommation en pommes et en biscuits } \\
\hline & $\begin{array}{l}\text { Pommes } \\
\text { en } \mathrm{g} \mathrm{kg}^{-1} \mathrm{j}^{-1}\end{array}$ & $\begin{array}{c}\text { Biscuits } \\
\text { en } \mathrm{g} \mathrm{kg}^{-1} \mathrm{j}^{-1}\end{array}$ \\
\hline $\begin{array}{c}\text { Régime } \\
\text { normal } \\
\mathrm{n}=5(5 \%)\end{array}$ & $345,5 \pm 23,2$ & $24,4 \pm 7,3$ \\
\hline $\begin{array}{c}\text { Régime } \\
\text { déshydraté } \\
n=4(4 \text { q) }\end{array}$ & 0 & $51,6 \pm 4,4$ \\
\hline
\end{tabular}


Les données expérimentales sur les flux d'entrée en sodium et en eau (tabl. 1 et 2) et les teneurs en sodium et en eau dans les aliments, ont permis de calculer les quantités de pommes et de biscuits ingérées par les mériones adultes (tabl. 3 ).

Les mériones soumises à une carence hydrique progressive dès leur sevrage consomment, lorsqu'elles sont adultes, deux fois plus de biscuits que les animaux témoins.

\section{Discussion.}

Les résultats obtenus montrent que, même lorsqu'elles sont en croissance, les mériones sont capables de résister à une carence hydrique prolongée imposée dès le sevrage. Comme la plupart des rongeurs semi-désertiques, la mérione se distingue à cet égard du rat chez lequel la carence hydrique, quelle que soit la façon dont elle est appliquée, provoque une perte de 40 à $50 \%$ en 5 à 6 jours, avec arrêt de prise alimentaire, ce qui le conduit à la mort (Katz et Arich, 1973). Cependant, parmi les $70 \%$ des mériones survivant à la carence hydrique, nous avons noté que dans un premier temps, les mériones déshydratées maintiennent sensiblement leur croissance tout en recevant un apport d'eau représentant la moitié ou le quart de leurs besoins. Lorsque les animaux sont soumis à une carence hydrique totale, l'eau disponible est essentiellement l'eau métabolique formée par le catabolisme des aliments. De façon à assurer les besoins minima, ils font sans doute appel à leurs réserves corporelles (lipidiques) et sont probablement encore incapables de restreindre suffisamment leurs pertes en eau, ce qui explique la chute de poids de $10 \%$. Cette hypothèse avait été avancée pour expliquer la perte de poids subie par des mériones adultes soumises à une carence hydrique (jusqu'à $12 \%$ de leur poids initial en trois semaines). Chez celles-ci, le poids se stabilise, ensuite les animaux survivent pendant plusieurs mois, mais sans jamais retrouver leur poids initial (Lachiver, communication personnelle). Chez les mériones en croissance, la stabilisation de poids (pendant environ 3 semaines) correspond probablement à une reprise d'appétit. Durant cette phase, la quantité de nourriture consommée serait suffisante pour maintenir les balances hydrique et énergétique de l'animal, mais non pour assurer sa croissance. C'est probablement pendant cette période que s'effectue la régulation des pertes en eau.

La reprise de poids enregistrée chez les mériones déshydratées, au bout des trois semaines de stabilisation, pourrait s'expliquer par une augmentation de la consommation alimentaire dès ce moment. Cette augmentation a été mise en évidence en fin d'expérience. A ce stade, l'animal utiliserait cet apport alimentaire pour fabriquer l'eau métabolique nécessaire à sa survie et à sa croissance. Son équilibre sodique est maintenu puisqu'à l'augmentation du flux entrant du sodium correspond une augmentation du même ordre du flux sortant, par rapport aux flux mesurés chez les témoins. De même, les mériones soumises à la carence hydrique réussissent à maintenir leur balance hydrique en diminuant du tiers leur flux sortant d'eau, par rapport aux témoins. Les taux de renouvellement de l'eau corporelle des mériones déshydratées sont significativement diminués par rapport à ceux des témoins. En effet certaines espèces vivant dans des régions désertiques survivent à une privation d'eau en diminuant considérablement leur taux de renouvellement d'eau. Chez la mérione adulte privée d'eau, celui-ci s'abaisse de 
$12 \%$ à $4 \%$ (Rouffignac et Morel, 1965). Les taux de renouvellement d'eau obtenus chez les mériones des deux régimes correspondent sensiblement à ceux déterminés, au laboratoire, par Bradshaw et al. (1976) sur deux groupes de Meriones shawi, l'un recevant un régime riche en eau, l'autre un régime pauvre en eau. Ces auteurs ont montré que pour un même régime alimentaire, composé de grains exclusivement, Meriones crassus, habitant les régions désertiques, a un taux de renouvellement d'eau plus élevé que celui de Meriones shawi, ce qui laisse supposer que $M$. crassus est plus adaptée à l'économie d'eau que $M$. shawi. Par ailleurs il a été montré que les rongeurs désertiques réduisent leurs pertes en eau en excrétant une urine très concentrée (Schmidt-Nielsen et Schmidt-Nielsen, 1961). L'hypertrophie rénale constatée chez les mériones déshydratées représenterait probablement un mécanisme compensateur permettant à l'animal de limiter ses pertes hydriques par une augmentation du pouvoir de concentration rénale. Ces résultats peuvent être corrélés à ceux obtenus chez la majorité des rongeurs désertiques excrétant une urine hyperosmotique par rapport au plasma, ce qui leur permet de maintenir un volume d'eau corporel constant (Schmidt-Nielsen et O'Dell, 1961). Dans une telle situation l'intervention du rein ne peut être négligée. Le pouvoir de concentration urinaire des rongeurs désertiques résulte d'un mécanisme physiologique extrêmement développé comparativement à celui d'autres espèces de mammifères non désertiques. Il a été décrit, en particulier, chez deux espèces de rongeurs australiens, Notomys alexis et Leggadina hermans burgensis (Mac Millen et Lee, 1967). Ainsi le rein, par sa grande capacité de concentration, joue un rôle important dans la conservation de l'eau corporelle. En effet, Hewitt (1981) constate, chez Notomys alexis, que le rein acquiert une certaine plasticité se traduisant par des modifications morphologiques et fonctionnelles et ceci dès le stade juvénile de l'animal (entre 11 et 23 jours). De même chez Mus musculus une déshydratation chronique imposée provoque une hypertrophie rénale se traduisant par une augmentation de $50 \%$ de l'épaisseur médullaire (Blount et Blount, 1968). D'ailleurs il existe chez les rongeurs désertiques, d'une façon générale, une corrélation entre la surface médullaire rénale, l'épaisseur médullaire et la concentration urinaire (Browfield et Wunder, 1976).

A la lumière de ces travaux, nous pensons que l'hypertrophie rénale des mériones déshydratées peut être le reflet de profondes modifications au sein de cet organe dont nous envisageons l'étude morphofonctionnelle.

Reçu en avril 1986.

Accepté en décembre 1986.

Remerciements. - Nous remercions Monsieur F. Petter (Laboratoire de Zoologie, Mammifères et Oiseaux du Muséum) pour ses précieux conseils, Monsieur C. Grizard pour l'iconographie, ainsi que Madame D. Goffinet. Ce travail a été effectué grâce au contrat A.T.P.-C.N.R.S., "Réponse biochimique et physiologique à des situations vitales critiques $n, n^{\circ} 960089$.

\section{Références}

BADDOURI K., BUTEN D., IMBERT-TEBOUL M., LE BOUFFANT F., MARCHETTI J., CHABARDES D., MOREL F., 1984. Plasma antidiuretic hormone levels and kidney responsiveness to vasopressin in the jerboa, Jaculus orientalis. Gen. comp. Endocrinol., 54, 203-215. 
BLOUNT R. F., BLOUNT I. H., 1968. Adaptive change in size of renal papilla with altered function. Tex. Rep. Biol. Med., 26, 473-484.

BRADSHAW D., CHENITI T., LACHIVER F., 1976. Taux de renouvellement d'eau et balance hydrique chez deux rongeurs désertiques, Meriones shawi et Meriones libycus étudiés dans leur environnement naturel en Tunisie. C. R. Acad. Sci., Paris, 282, sér. D, 481-484.

BROWFIELD M. S., WUNDER B. A., 1976. Relative medullary area; a new structural index for estimating urinary concentrating capacity in mammals. Comp. Biochem. Physiol., 55A, 6975.

GREEN B., 1978. Estimation of food consumption in the dingo, Canis familiaris dingo, by means of $22 \mathrm{Na}$ turnover. Ecology, 59, $\mathrm{n}^{\circ} 2,207-210$.

HEWITT S., 1981. Plasticity of renal function in the australian desert rodent Notomys alexis. Comp. Biochem. Physiol., 69A, 297-304.

IVANOVA L. N., NATOCHIN YU. V., SEREBRYAKOV E. P., GONCHAREVSKAYA O. A., KNYAZKOVA L. G., LAVROVA E. A., NASLEDOVA N. I., PECHURKINA N. I., PODSEKAEVA G. V., SHAKHMATOVA E. I., 1980. Comparative study of the concentrating mechanism in the kidney of the big gerbil (Rhombomys opimus L.) and the water role (Arvicola terrestris L.). Comp. Biochem. Physiol., 66A, 499-505.

KATZ U., ARICH B., 1973. Mechanical activity of the gastrochemius muscle in the albino rat under water deprivation at different temperatures. Comp. Biochem. Physiol., 44A, 1387-1395.

LACHIVER F., CHENITI T., BRADSHAW D., BERTHIER J. L., PETTER F., 1978. Field studies in southern Tunisia on water turnover and thyroidactivity in two species of meriones, 81-84. In ASSENMACHER Y., FARNER S., Environmental endocrinology, Springer-Verlag.

LIFSON N., MC CLINTOCK R., 1966. Theory of use of the turnover rates of body water for measuring energy and material balance. J. theoret. Biol., 12, 46-74.

Mac MILLEN R. E., LEE A. K., 1967. Australian desert mice: independence of exogenous water. Science, N. Y., 158, 383-514.

MORE T., SAHNI L. K., 1979. Effects of water deprivation on growth and body fiuid composition in chokla lambs under semi arid conditions. Indian J. anim. Sci., 48, 600-604.

NAGY K. A., COSTA D. P., 1980. Water flux in animals: analysis of potential errors in the tritiated water method. Am. J. Physiol, 238, R454-R465.

PETTER F., 1961. Répartition géographique et écologique des rongeurs désertiques de la région Paléarctique. Thèse Doct. ès Sci. Nat. Fac. Sci. Paris. Mammalia, 25, 1-222.

PETTER F., LACHIVER F., 1983. Les adaptations des rongeurs Gerbillidés à la vie dans les régions arides. Coll. Bioclimatologie méditerranéenne, Colloque associé au C.N.R.S., mai, 18-20.

PUROHIT G. R., GHOSH P. K., TANEJA G. C., 1972. Water metabolism in desert sheep : effect of various degrees of water restriction on the distribution of body water in Marwari sheep. Aust. J. agric. Res., 23, 605-691.

ROUFFIGNAC C., MOREL F., 1965. Etude comparée du renouvellement de l'eau chez quatre espèces de rongeurs, dont deux espèces d'habitat désertique. J. Physiol, Paris, 58, $309-322$.

SCHMIDT-NIELSEN K., 1964. Desert animals : physiological problems of heat and water. Clarendon Press, Oxford ; Dover Publ., New York, 277 pp.

SCHMIDT-NIELSEN B., O'DELL R., 1961. Structure and concentrating mechanism in the mammalian kidney. Am. J. Physiol., 200, 1119-1124.

SCHMIDT-NIELSEN K., SCHMIDT-NIELSEN B., 1961. Water metabolism of desert mammals. Physiol. Rev., 32, 135-166.

TANEJA G. C., 1965. Effects of varying frequency of watering during summer on the temperature respiration body weight and packed cell volume of blood of sheep. Ind. J. exp. Biol., 4, 259. 262.

ZNARI M., 1983. L'utilisation du sodium 22 (22 Na) et de l'eau tritiée ( 3 HHO/ pour l'estimation de la consommation alimentaire et des besoins hydriques chez deux espèces de rongeurs: Acomys airensis (Muridae), Meriones unguicularus (Gerbillidae). Diplôme de D.E.A. d'Ecologie, Fac. Sci. Paris VI, 1-41. 